\title{
La educación en J.J. Rousseau: un antecedente metodológico de la enseñanza basada en la formación en competencias
}

\section{Education in J.J. Rousseau: a methodological antecedent of competence based teaching}

\author{
Beatriz SIERRA-ARIZMENDIARRIETA y Miguel PÉREZ-FERRA \\ Universidad de Oviedo y Universidad de Jaén
}

Recibido: Junio 2013

Aceptado: Septiembre 2013

\begin{abstract}
Resumen
Sabiendo la enorme repercusión e influencia que ha tenido J.J. Rousseau en la pedagogía moderna, el reciente tricentenario de su nacimiento puede ser una buena oportunidad para reflexionar sobre su extraordinaria actualidad. El presente trabajo es una investigación teóricoeducativa llevada a cabo con un método hermenéutico analítico-comparativo. Su objetivo es mostrar cómo algunos planteamientos de su pensamiento educativo poseen gran similitud con ciertos cambios que exige la actual enseñanza basada en la formación en competencias, de modo que podría considerarse como un antecedente metodológico de la misma. Para lograr este objetivo se ha dividido la exposición en tres partes. En la primera parte se realizará un análisis de la teoría educativa de Rousseau en los tres primeros libros del Emilio, en los que uno de los temas principales es el aprendizaje basado en la propia experiencia, fomentando la autosuficiencia, la curiosidad y la motivación por aprender. Rousseau propone como método la educación negativa que exige, entre otras cosas, un seguimiento constante del aprendiz por parte del tutor. En la segunda parte se abordará una breve exposición de los cambios más relevantes y características de la enseñanza basada en la formación en competencias, así como su finalidad. Destaca la necesaria actividad y participación del alumnado en su propio aprendizaje a través de la realización de tareas. El nuevo modelo educativo lleva consigo un cambio radical en la estructura curricular en el que destaca la transformación de la metodología de aula y el rol docente. Para finalizar, la tercera parte tendrá como objetivo elaborar una síntesis comparativa entre ambas propuestas agrupando los paralelismos en cuatro temas: el origen de ambos modelos, su finalidad, metodología y cambio en la función docente.
\end{abstract}

Palabras clave: método activo, aprendizaje por experiencia, rol del profesor, competencias.

\begin{abstract}
Taking into account the huge repercussion and influence that J.J. Rousseau has had on modern pedagogy, the recent tercentenary of his birth is a good opportunity to think about his outstanding relevance nowadays. This paper is a theoretical and educative research developed with an analytic and comparative hermeneutical method. The main objective is to show how some concepts of his philosophy of education have a great similarity with certain changes that
\end{abstract}


the present competency based teaching is demanding, so it could be considered its methodological background. In order to achieve this objective this exposure has been divided in three parts. The first part is an analysis of Rousseau's educational theory as developed in the first three books of the Emilio, in which one of the main themes is self experience-based learning, fostering self-sufficiency, curiosity and the motivation for learning. Rousseau proposed as a method the negative education, which requires, among other conditions, a constant monitoring of the learner by the tutor. In the second part, a brief summary of the most relevant changes and characteristics of competency-based teaching is developed, as well as its purpose.

The student's participation and activity are highlighted within their own learning process through the carrying out of tasks. The new educational model involves a radical change in the curriculum, in which it is highlighted the transformation of the methodology used in the classroom as well as the role of the teacher. Finally, the aim of the third part is to offer a comparative synthesis of both proposals grouping the parallelisms found in 4 topics: origin of the two models, its aims, methodology, and change in the teaching roles.

Keywords: active method, learning from experience, teacher role, competencies.

\section{Planteamiento y objetivo del trabajo}

El 28 de junio de 2012 se cumplió el tricentenario del nacimiento de Jean-Jacques Rousseau. Es bien conocida la influencia de sus ideas en la educación contemporánea, en la que se ha situado al alumno en el centro del proceso de enseñanza-aprendizaje y destacado su actividad. Por ello, es considerado por muchos autores como el precursor de la pedagogía moderna (Gutiérrez Zuloaga, 2002; Oelkers, 2002; Wendt y Dalbosco, 2012). Existen varios enfoques o tradiciones de investigación, dentro de los cuales podemos encontrar diferentes definiciones del concepto de competencia: el enfoque conductista o behaviorista, el enfoque genérico y el enfoque cognitivo (Mulder, Weigel y Collings, 2008). Aunque hay una multiplicidad de concepciones de competencia, consideramos dos modelos dominantes: un primer modelo, que define el término competencia de manera individual y cognitiva (de origen anglosajón); un segundo modelo, posterior, promovido por la sociología francesa, que pone un mayor énfasis en el componente social de la competencia. Desde esta concepción de competencia, en la que se sitúan autores como Roegiers o Perrenoud, entre otros, adscribimos nuestra percepción del concepto.

Este artículo aborda las evidencias que ponen de manifiesto la vigencia de la pedagogía rousseauniana, tratando de mostrar el paralelismo existente entre ella y el modelo socioconstructivista -ligado al enfoque cognitivo, pero situado-, presente en diversos ordenamientos educativos, para formar en competencias. Sus antecedentes inmediatos se hallan en la asunción que la Unión Europea ha hecho del proyecto DeSeCo (Definición y Selección de Competencias), auspiciado por la OCDE (2005), cuya finalidad es capacitar al alumno para utilizar eficazmente el conocimiento adquirido mediante un aprendizaje situado. 
Entendemos que la formación en competencias representa: "La capacidad de movilizar recursos para poder hacer, pero no entendida como mera aplicación, ni como una reconstrucción, sino como un saber hacer con valor añadido, el que le aporta la singularidad de cada persona" (Pérez Ferra, 2011, p. 78). Recientes estudios han profundizado en la definición e interpretación de las competencias básicas en Europa (Escamilla, 2008; Halász y Michel, 2011), han analizado la evaluación de las competencias básicas en el currículo (Dam, Geijsel, Reumerman y Ledoux, 2011; Pepper, 2011) o han reflexionado sobre su implementación (Goñi, 2009; Tiana, Moya y Luengo, 2011). El informe internacional de Gordon et ál. (2009) reconoce que los profesores son los principales actores en el cambio hacia las competencias y sostiene que la implementación del enfoque basado en competencias depende fundamentalmente de su actitud. Pero son aún escasas las investigaciones centradas en la percepción docente (Méndez-Giménez, Sierra-Arizmendiarrieta y MañanaRodríguez, 2013). Dada la limitación del espacio, este trabajo no pretende realizar una defensa o crítica de este modelo formativo, sino que únicamente tiene como objetivo mostrar un paralelismo entre algunos rasgos que parecen esenciales de la enseñanza que forma en competencias - con una orientación socioconstructivista-, y la pedagogía de Rousseau.

El objeto de análisis serán los tres primeros libros del Emilio, cuyo objetivo es mostrar que la propuesta rousseauniana anticipa lo que constituye el principal cambio de metodología en la enseñanza actual, que se centra en la práctica del alumnado y en la resolución de problemas como forma de adquirir y desarrollar competencias, ayudando al que se educa a hacerse progresivamente más autónomo, en relación con el mundo y con los demás. La finalidad de su planteamiento es lo que sirve de enlace entre esos tres primeros libros y los dos últimos, así como con el contenido del Contrato Social, pero nuestro interés se ha centrado exclusivamente en su proyecto para las primeras edades del aprendizaje, dejando también al margen del análisis el resto de su obra, tanto política como autobiográfica. Se divide el trabajo en tres partes: 1) análisis y recopilación de algunas referencias principales de la teoría educativa rousseauniana en los tres primeros libros del Emilio; 2) breve exposición de los cambios más relevantes, características y finalidad de la enseñanza basada en competencias; 3) síntesis comparativa, a modo de conclusión, entre la propuesta metodológica del ginebrino y las exigencias derivadas de la actual enseñanza basada en la formación en competencias.

La novedad de este trabajo no radica, por tanto, en la mejora de la comprensión de la pedagogía de Rousseau, ni en un avance o posicionamiento en el complejo debate conceptual sobre la enseñanza basada en la formación en competencias. La intención última es ofrecer un nuevo antecedente a este debate, pudiendo situar el enfoque socioconstructivista de la formación en competencias como heredero de una tradición que sitúa al alumno en el centro del proceso de enseñanza-aprendizaje. Ello pone nuevamente de actualidad algunas de las propuestas del ginebrino. 


\section{La educación en el Emilio: un aprendizaje basado en la experiencia}

Los escritos educativos de Rousseau están ligados a sus ideas políticas y tienen un objetivo claro: mostrar cómo se puede ser libre en sociedad. Así, su finalidad se encuentra ligada a un compromiso ético individual y social. Su filosofía tiene su origen en la crítica a la sociedad existente por considerar que no permite la libertad. En el Emilio realiza una crítica al método pedagógico de sus días (Arrocha González, 2000). Kontio ve la solución a la contradicción que Rousseau plantea entre la naturaleza y la sociedad, en la "autarquía" (Kontio, 2003, p. 17) que propone en el Emilio, como el equilibrio entre las necesidades humanas y la capacidad para solventarlas. En estas páginas, se hablará de 'autosuficiencia' para designar dicho equilibrio en el ámbito físico, dejando el término de 'autonomía' para el ámbito moral (Sierra y Arizmendiarrieta, 1997).

Hasta el libro III Rousseau considera a Emilio sólo como un ser físico (OC, IV, p. $458)^{1}$. Su dimensión moral se inicia cuando comienza su relación con los otros (ya en el libro IV) y su amour propre, que habrá de ser encauzado con la virtud. Sabiendo que cada etapa ha de considerarse sólo como un medio para el perfeccionamiento de Emilio en las siguientes fases, nos centraremos, exclusivamente, en sus indicaciones en los tres primeros libros para aprender a desenvolverse en el mundo, logrando una autosuficiencia como expresión de la máxima libertad. Esta etapa termina cuando Emilio cumple quince años.

Rousseau aconseja tener una única guía o referente: "observad la naturaleza y seguid la ruta que ella os traza" (OC, IV, p. 259). Su teoría educativa se basa en encauzar el desarrollo natural del niño sin anticipar etapas, de manera que vaya aprendiendo solo, pues únicamente así será capaz de llegar a ser libre y bastarse a sí mismo en la edad adulta, en sociedad, no teniendo "más necesidad de otro guía que él mismo" (OC, IV, p. 264). La educación en Rousseau ha de ser una especie de "desnaturalización natural” (Burgelin, 1952, p. 484), elevándolo por encima de la naturaleza, pero sin oponerse a ella, siguiendo su dinámica propia. Para Rousseau ello exige una vigilancia extrema y constante. A este respecto, es interesante la distinción que establece Hannoun (1979) entre una pedagogía espontaneísta, que consiste en dejar hacer a la naturaleza, y la que toma la naturaleza por modelo. Rousseau persigue esto último.

La necesidad de actuar por uno mismo y, a partir de ahí, el planteamiento de un aprendizaje básicamente experiencial, será el tema central de los tres primeros libros. No se analizarán los dos siguientes, pues en ellos se adentra en el carácter moral en la educación y la futura vida en sociedad, cobrando mayor importancia el compromiso ético con la adquisición de la virtud y la búsqueda del bien común.

\footnotetext{
${ }^{1}$ Las referencias a la obra de Rousseau se van a citar -por ser la edición oficial de referenciasegún las Oeuvres Complêtes $(O C)$, en cinco volúmenes, publicadas por Bernard Gagnebin y Marcel Raymond, en la Bibliothêque de la Pléiade, Paris, Gallimard. De ahí que las referencias a su obra en el cuerpo del texto aparecerán como $(O C$, vol, p.).
} 


\section{Libro I:}

Rousseau defiende que nada de lo que se haga en la educación de un niño es indiferente o neutro. Critica el modo usual de cuidar a los niños desde su nacimiento y resalta el hecho de que un mal cuidado físico puede derivar en ideas morales inapropiadas. Por eso, establece un paralelismo entre el encorsetamiento físico de un recién nacido y la esclavitud en que vivirá a causa de las instituciones (OC, IV, 253). En el inicio de la educación se deberá apostar por la libertad de movimientos, como un primer paso hacia la autosuficiencia. Rousseau propone que desde el comienzo aprendan a limitar sus deseos a sus fuerzas, ofreciendo cuatro máximas para orientar los primeros cuidados: 1) dejarles usar todas sus fuerzas; 2) ayudarles en lo que les falte, en lo relativo a las necesidades físicas; 3) limitarse a la utilidad real, sin conceder caprichos; 4) estudiar su lenguaje y signos para saber distinguir entre sus necesidades y sus caprichos (OC, IV, 290).

Tiene una especial relevancia la importancia que otorga al aprendizaje por la experiencia. Será una constante unida a la utilidad. Emilio aprenderá manipulando: "quiere tocarlo todo, manejarlo todo: no os opongáis a esta inquietud; ella le suscita un aprendizaje muy necesario" (OC, IV, p. 284). El niño sólo se aplicará en aquello que le suscite un interés por la utilidad que le proporciona: "él sabrá hablar por sí mismo, a medida que sienta la utilidad de hacerlo" (OC, IV, p. 297).

Al final del libro I afirma que, en los primeros años de la infancia, el niño aprende casi simultáneamente a comer, a andar y a hablar, pero carece de sentimientos e ideas, y tiene aún pocas sensaciones. Partiendo de ese convencimiento, Rousseau basará su educación 'natural' en ayudar a Emilio a desarrollar los órganos y educar sus sentidos (libro II), para posteriormente adquirir de forma adecuada sentimientos e ideas, junto con el juicio (libro III).

\section{Libro II:}

Este libro trata de la educación de Emilio en lo que Rousseau llama el "segundo término de la vida" (OC, IV, p. 299) que abarca aproximadamente entre los cinco y los doce años. De igual forma que en el libro I, Rousseau insiste en que los niños deben aprender por sí mismos, oponiéndose a la práctica habitual de la educación de su tiempo: "Nuestra manía magistral y pedantesca es siempre la de enseñar a los niños cuanto ellos aprenderían mucho mejor por sí mismos" (OC, IV, p. 300). Defiende que el aprendizaje debe provenir de la experiencia, e incluso llega a afirmar que es mejor ignorar algo a necesitar que otro se lo facilite (OC, IV, 404). Uno sólo aprende lo que ha experimentado. Añade también una primera crítica a las enseñanzas verbales, apostando por las acciones frente a los discursos (OC, IV, 321, 328, 333) a los que también atacará en el libro III: "yo no estimo las explicaciones por medio de discursos; los jóvenes les prestan poca atención y no los retienen” (OC, IV, p. 447).

En la educación tradicional, el alumno no era considerado como el sujeto del proceso pedagógico, sino como receptáculo de informaciones. Wendt y Dalbosco (2012) destacan dos grandes cambios que realiza Rousseau respecto a la educación hasta ese momento: por una parte, su idea de la educación por las cosas, en la que la razón no se encuentra en el inicio del proceso educativo y, por otra parte, su concepto 
de infancia, proponiendo considerar las capacidades de cada edad como punto de partida del proceso pedagógico. A este respecto, pueden encontrarse muestras de la importancia que Rousseau otorga a cada etapa del niño (OC, IV, 241-242; 418), lo que influirá posteriormente en Piaget (Oelkers, 2002). Refiriéndose ya explícitamente a la infancia, opina que hay que respetarla en el orden de la vida humana (OC, IV, 303, $343)$, puesto que la infancia tiene modos de ver, pensar o sentir propios (OC, IV, 319), achacando los errores y las consecuencias negativas de una educación equivocada a que "se les quiere hacer hombres antes de tiempo" (OC, IV, p. 372).

En la Carta a Christophe de Beaumont habla de lo que llama "educación positiva" que consiste en "formar el espíritu antes de la edad (adecuada) y a inculcar al niño el conocimiento de los deberes del hombre" (OC, IV, p. 945). La educación positiva sería aquella que no respeta las capacidades de cada etapa del niño, enseñándole cosas que no entiende o para las que no se encuentra preparado. Rousseau, en el segundo libro, defiende que "antes de la edad de la razón, el niño no recibe ideas, sino imágenes" (OC, IV, p. 344). Esta edad de la razón comienza más tarde, hacia los quince años, aunque afirma que antes posee una especie de razonamiento que se relaciona con su interés presente y sensible (OC, IV, 345), que llama razón sensitiva ${ }^{2}$. Ésta forma ideas simples a partir de sensaciones y la distingue de la razón intelectual, que forma ideas complejas a partir de ideas simples (OC, IV, 370, 417).

En la edad descrita, que en la actualidad corresponde a la Educación Primaria, Rousseau defiende que los niños están en una edad de la sensación. Puesto que sólo retienen sensaciones, carecen también de memoria (OC, IV, 350), por lo que lo primero que hay que cultivar son los sentidos (OC, IV, 380) y a ello dedica buena parte del libro II, en el que habla de cada uno y propone varios ejemplos para su desarrollo mediante la experiencia (OC, IV, 388-417). Los sentidos y los órganos a ellos asociados sólo pueden desarrollarse mediante el ejercicio (OC, IV, 402) y la práctica continuada. A este respecto abordamos la interpretación de la epistemología rousseauniana a través de tres etapas de desarrollo: el conocimiento originado en las impresiones sensoriales, la transición de la sensación a la capacidad de juicio, y su concepción del desarrollo mental, indispensable para la virtud ética (Hanley, 2012).

Para Rousseau, la única metodología posible en los primeros años es la que denomina "educación negativa". "La primera educación debe ser, pues, puramente negativa. Consiste, no en enseñar la virtud ni la verdad, sino en defender al corazón del vicio y del espíritu del error" (OC, IV, p. 323). Rousseau completa en qué consiste este tipo de educación en la Carta a Christophe de Beaumont (OC, IV):

Llamo educación negativa a la que tiende a perfeccionar los órganos, instrumentos de nuestros conocimientos, antes de darnos estos conocimientos, y que prepara la razón por el ejercicio de los sentidos. La educación negativa no es ociosa, ni mucho menos. No inculca las virtudes, pero previene los vicios; no enseña la verdad, pero preserva del

\footnotetext{
${ }^{2}$ La denominada por Rousseau 'razón sensitiva' constituye el antecedente de la nueva teoría de las emociones de finales del siglo XX, siendo muy avanzada para su época (Camarero, 2010). 
error. Dispone al niño en todo cuanto puede conducirle a la verdad cuando esté en condiciones de entenderla, y al bien cuando esté en condiciones de amarlo (p. 945).

La educación negativa es, así, una suerte de preparación del terreno para que la semilla pueda fructificar. Rousseau en el Emilio la llama también "método inactivo" (OC, IV, p. 359), porque no lo entiende como una enseñanza activa, en la que el educador tenga que hacer o enseñar algo, sino más bien evitar todo lo negativo. Se podría decir que es una educación preventiva. Supone un cambio en el rol del educador de manera que no ha de preocuparse en 'añadir' conocimientos, sino en evitar efectos negativos o perjudiciales en las distintas experiencias del alumno.

Rousseau aconseja: "Joven preceptor, os predico un arte difícil, y éste es el de dirigir sin preceptos y hacerlo todo no haciendo nada" (OC, IV, p. 362). Dirigir sin preceptos supone ser capaz de guiar al alumno sin que éste se dé apenas cuenta, y anticiparse a cualquier cosa que pueda querer o hacer, lo que exige también un conocimiento en profundidad del mismo. Requiere una constante atención y, al mismo tiempo, orientar y dirigir las potencialidades que le ofrece la naturaleza para aprovecharlas. Rousseau afirma que Emilio "no debe hacer más que lo que quiera; pero no debe querer sino lo que queráis que haga; no debe dar un paso que no lo hayáis previsto, no debe abrir la boca sin que sepáis lo que va a decir" (OC, IV, p. 363). La educación es, así, sinónimo de previsión, no pudiendo dejar nada al azar, lo que exige que, en ocasiones, haya que manipular o provocar situaciones de las que se obtenga un aprendizaje, teniendo en cuenta que la más importante y útil regla de la educación "no es ganar tiempo, sino perderlo" (OC, IV, p. 323).

Dado que el aprendizaje corre por cuenta del niño a partir de las distintas situaciones que se organicen a tal efecto, lo más importante para facilitar su aprendizaje es provocar el "deseo de aprender" (OC, IV, p. 358), sabiendo plantear ante el niño situaciones en las que necesite aprender algo para resolver un problema o lograr lo que desea (OC, IV, 394-403). El aprendizaje ha de estar enfocado a todo aquello que sea útil para poder conducirse en la vida. Como ejemplo, afirma: "Es necesario que él sepa leer cuando la lectura le sea útil” (OC, IV, p. 357). Así se mejorará también la atención (OC, IV, 387), puesto que no se puede ejercitar si no va unida al interés. Igualmente, Rousseau critica la enseñanza de su tiempo, el enseñar las cosas directamente, sin dar oportunidad a la experiencia. Se opone a un ejercicio memorístico sin sentido para el niño, con el que no aprende a razonar: "en lugar de hacernos buscar las demostraciones, se nos dictan; que en lugar de enseñarnos a razonar, razona por nosotros el profesor y no ejercita, nada más que nuestra memoria" (OC, IV, p. 399).

Rousseau no está en contra del uso de la memoria en el aprendizaje, sino de facilitarle el conocimiento 'ya hecho'; por el contrario, todo lo que aprenda por sí mismo a partir de la utilidad que le presta, se le quedará grabado con facilidad y le servirá de por vida (OC, IV, 351).

El mismo método será empleado en el libro III, aunque hace su aparición la formación de la razón intelectual como continuidad de la sensitiva. Hacia el final del 
libro II anuncia que tratará de un sexto sentido, el sentido común, ya relacionado con las ideas (OC, IV, 417).

\section{Libro III:}

Este libro aborda "el tercer estado de la infancia" (OC, IV, p. 426), y abarca aproximadamente de los doce a los quince años. Rousseau explica que hasta aquí ha velado por la actividad del cuerpo (a través del desarrollo de los sentidos) y que ahora es necesario ocuparse en "la actividad del espíritu que busca instruirse" (OC, IV, p. 429).

La noción de utilidad se convierte ya en algo prioritario. "No se trata de saber todo, sino solamente lo que es útil" (OC, IV, p. 428). Así, excluye los conocimientos especulativos como poco apropiados a la edad, debiendo ocuparse con "una aplicación más constante" en los "objetos de utilidad real" (OC, IV, p. 444), teniendo en cuenta que la utilidad es relativa y ha de adaptarse a la edad (OC, IV, 445). El método empleado continúa siendo la educación negativa, pues "lo único funesto es el error" (OC, IV, p. 428), siendo más importante no caer en un error que saber la verdad de las cosas (OC, IV , 485). "El mal no está en lo que él no entiende, sino en lo que cree entender" (OC, IV, p. 451). El error procede del juicio (OC, IV, 483), por eso tiene gran importancia enseñar a juzgar bien. Rousseau explica cómo en la sensación el juicio es pasivo, convirtiéndose activo en la percepción o idea que es quien reúne y compara las sensaciones (OC, IV, 481). Lo más importante para enseñar a no equivocarse no es enseñar una verdad, sino demostrar "cómo es necesario proceder para descubrir siempre la verdad" (OC, IV, p. 484). Rousseau identifica el arte de juzgar con el arte de razonar (OC, IV, p. 486).

Con la aparición del juicio o del razonamiento, las referencias al aprendizaje autónomo son aún mayores que en el libro II. Emilio no debe aceptar lo que no comprenda y ha de aprender partiendo de su propia experiencia. Esta razón pedagógica se basa en un principio simple: "no se comprende bien lo que no se aprende por sí mismo, no se aprende por sí mismo lo que no se tiene interés en aprender" (Guénard, 2009, p. 17). Es especialmente significativo el texto siguiente del libro III (OC, IV):

Haciendo que vuestro alumno esté atento a los fenómenos de la naturaleza, bien pronto le haréis curioso; pero para nutrir su curiosidad, no os apresuréis nunca a satisfacerla. Poned las cuestiones a su alcance, y dejadle resolverlas. Que él no sepa nada porque se lo hayáis dicho, sino porque lo haya comprendido por sí mismo; que no aprenda la ciencia, que la invente. Si nunca sustituís en su espiritu la autoridad a la razón, él no razonará jamás; únicamente será el juguete de la opinión de los demás (p. 430).

Como se ha indicado al comienzo, una de las cosas que más le preocupa a Rousseau es la falta de libertad. Su pensamiento educativo es un medio de mostrar cómo ser más libre, teniendo en cuenta la debilidad humana física y moral. Para lograrlo, Emilio ha de ser capaz de no depender de la opinión ajena. Ésta es la finalidad última que lleva a Rousseau a plantear un método en el que la propia experiencia esté por encima de una 
exposición verbal sin referente claro para el que aprende. Antes de que se hablara del socioconstructivismo y del aprendizaje significativo, Rousseau ya advierte que aprender algo que no se comprende es inútil y no facilita la independencia del aprendiz. Insistiendo en la necesidad de ejercitarse y adquirir práctica afirma que "una hora de trabajo le enseñará más cosas que él retendría en un día de explicaciones" (OC, IV, p. 456). Al sustituir la lección por la experiencia, Rousseau no puede estar más alejado de una pedagogía transmisiva y más cerca de los métodos activos constructivistas (Fedi, 2011).

La educación negativa supone un cambio en el rol docente. La independencia en el aprendizaje, que propone para Emilio, necesita supervisión, guía sin que lo advierta (OC, IV , 435). Esa guía imperceptible es consecuencia de su máxima del libro II, ya citada, cuando propugna el "dirigir sin preceptos y hacerlo todo no haciendo nada" (OC, IV, p. 362). De acuerdo con este método, el difícil trabajo del educador lleva implícito principalmente:

- Estimular, motivar al aprendiz para despertar su interés y gusto por el aprendizaje: "No se trata de enseñarle las ciencias, sino de darle el gusto para amarlas y métodos para aprenderlas, cuando ese gusto esté mejor desarrollado" (OC, IV, p. 436). Por ello, advierte que no se debe nunca obligarle, sino que ha de aprender por placer para poder mantener la atención. No se trata de motivarle con premios, sino ayudarle a encontrar una motivación intrínseca, pues, como algunos destacan, la motivación para Rousseau "viene de dentro" (Romani y Rajobac, 2011, p. 106).

- Mostrarle los caminos para que él mismo los recorra: "Le muestro la ruta de la ciencia, acomodada hacia la verdad, pero larga, inmensa, lenta de recorrer (...). Forzado a aprender por sí mismo, él usa de su razón y no de la de los demás" (OC, IV, p. 486).

- Situar a su alcance, sin imponerlo, un objeto de interés y los medios para alcanzarlo: "Es muy raro para vosotros proponerle aquello que él deba aprender: está en él desearlo, buscarlo, encontrarlo; a vosotros os corresponde situarlo a su alcance, hacer que nazca hábilmente ese deseo y facilitarle los medios para satisfacerlo" (OC, IV, p. 447). Aunque el tutor ha de actuar muy discretamente, sin su intervención el aprendizaje no sería posible. Ha de tener muy claro un proyecto y saber aprovechar las circunstancias (Simões Francisco, 2000).

Estas acciones han de concebirse de forma integrada, pues constituyen distintas fases entrelazadas de la misma tarea de vigilancia del aprendiz, por el tutor, y un exhaustivo conocimiento de su carácter, capacidades e intereses. Lo que facilita la labor de guía. Resulta interesante ver la similitud de lo manifestado por Rousseau acerca del papel del docente con lo que escriben Ausubel, Novak y Hanesian: "El papel más importante y distintivo del profesor en el salón de clase moderno, es el de ser director de las actividades de aprendizaje. Al dirigir las actividades de aprendizaje de los alumnos, la principal función del profesor ya no es, o ya no debiera ser, la de darles información" (1983, p. 432). Es preciso advertir que los citados autores no están en contra de la transmisión de información, que afirman ser necesaria, pero puede ser suministrada a través de diversos materiales. Rousseau escribe en el Emilio en contra 
de la pedagogía transmisiva, aunque su crítica se sitúa en la edad infantil en la que piensa que falta experiencia y referentes para la comprensión de ciertos contenidos. En lo que hay plena coincidencia entre Rousseau y Ausubel es en considerar la necesidad de un cambio en la función docente, dirigiendo las tareas o situaciones de aprendizaje y otorgando al alumno un papel más activo en la comprensión y significatividad de su aprendizaje.

\section{Algunas características, finalidades y cambios que aporta la formación en competencias}

La incorporación de las competencias a la formación, plantea la superación de una enseñanza memorística que lleva implícita la dificultad de aplicar los conocimientos a la vida real (Zabala y Arnau, 2007); además de evitar la saturación de contenidos en los currículos, que aun siendo relevantes, su inflación impide un aprendizaje significativo. Es necesario advertir que el aprendizaje memorístico que pretende evitar la formación en competencias, de la que aquí hablamos, es únicamente de tipo repetitivo y sin sentido para el que aprende, dejando, por tanto, a salvo la reivindicación de la necesidad de una memoria comprensiva.

La función educativa de las competencias se orienta al logro de la progresiva autonomía de los estudiantes, el ejercicio de la ciudadanía y la aportación de la formación al servicio de la comunidad. Sánchez Santamaría (2012) ha afirmado:

El término competencia básica queda articulado en torno a un conjunto de saberes cognitivos, procedimentales y actitudinales que pueden y deben ser alcanzadas a lo largo de la educación obligatoria por todo el alumnado y que resultan imprescindibles para garantizar el desarrollo personal y social y la adecuación a las necesidades que presente su contexto, así como para el ejercicio efectivo de los derechos y deberes del ciudadano (p. 132).

Desde hace más de dos décadas, nuestro sistema educativo ha destacado la necesaria actividad y participación del alumnado en su propio aprendizaje, aspecto imprescindible para abordar la habilitación de los alumnos en competencias básicas. La actividad y participación que supone el desarrollo de competencias pretende facilitar un aprendizaje más significativo $\mathrm{y}$, como consecuencia, lograr una motivación mayor al advertir el propio estudiante un avance en su conocimiento. El nuevo modelo educativo lleva consigo un cambio radical en la estructura curricular, que afecta fundamentalmente a dos aspectos:

- A la metodología: El aprendizaje es eminentemente práctico, adquiriendo especial importancia las tareas (Domingo Segovia y Barrero Fernández, 2010; Rodríguez Torres, 2010; Viso Alonso, 2010). La estructura y el contexto de las tareas constituyen el núcleo del aprendizaje. "Las tareas configuran situaciones-problema que cada estudiante debe tratar de resolver haciendo un uso adecuado de los contenidos escolares" (Tiana, Moya y Luengo, 2011, p. 317), de manera que funcionan como "microcontextos de aprendizaje" (Moya Otero, 2008, p. 73). Aprender partiendo de situaciones problemáticas tiene, a su vez, como 
consecuencia que los contenidos se entiendan de forma interrelacionada, facilitando un método más globalizado, que plantee mayor conexión con el contexto vital de los estudiantes.

- Al rol docente: que será más facilitador y orientador. "Como facilitador, el docente no sólo tendrá que presentar los contenidos, sino conducir a los discentes a través de diferentes caminos que comuniquen unos contenidos con otros, recorriendo distintos trayectos que permitan una visión más amplia y profunda del mapa de contenidos" (Sierra y Arizmendiarrieta; Méndez-Giménez; Mañana-Rodríguez, 2013 , p. 177). Esto requiere apostar también por la práctica reflexiva como una clave del nuevo rol docente (Perrenoud, 2004).

Abordados los referentes metodológicos, resulta procedente aludir a la morfología de las competencias, que han de trabajarse aproximadamente en el mismo periodo que Rousseau denomina infancia. Es interesante, por su sistematicidad, tener en cuenta las características planteadas por Viso Alonso (2010), aunque su valoración queda completada con otras apreciaciones:

- Carácter holístico/integrador. Ser competente integra y relaciona atributos personales, demandas externas y contexto. Es, por tanto, más que un simple conjunto de habilidades técnicas, de manera que supone tanto una interpretación de la realidad como una respuesta que damos de acuerdo con ella. Por eso, la competencia empieza, antes que en la acción para solventar el problema, en la definición adecuada del mismo.

- Carácter contextual. La enseñanza que habilita para adquirir competencias está situada, asociada a un determinado contexto y situación, pero el ejercicio de la competencia adquirida se puede extrapolar a diversas situaciones y momentos. Por tanto, el reto del aprendizaje es desplegar la potencialidad de lo aprendido en la resolución de problemas nuevos o en la capacidad de desenvolverse en una situación inesperada.

- Carácter transferible. Los problemas reales exigen visión de conjunto y movilización de recursos (tanto materiales como intelectuales). Por tanto, no basta con aprender a partir de un contexto, sino que es preciso saber extraer lo común de situaciones similares para adaptar el conocimiento a lo nuevo, así como saber distinguir entre situaciones similares y dispares. El aprendizaje no está orientado a 'demostrar' el dominio de unos contenidos con los que quizá no se sabe qué hacer, sino a saber desenvolverse en distintas situaciones de forma eficaz.

- Carácter reflexivo y creativo. Cada situación es única y requiere una solución a la medida, nueva, no reproduciendo mecánicamente una técnica o protocolo. Esto exige reflexión y acción. En el documento elaborado por la OCDE sobre la definición y selección de competencias clave, se considera la reflexión como el "corazón de las competencias clave" (OCDE, 2005, p. 7), requiriendo que "los individuos alcancen un nivel de madurez social que les permita distanciarse de las presiones sociales, adoptar diferentes perspectivas, hacer juicios independientes y tomar responsabilidad por sus acciones" (OCDE, 2005, p. 8). 
- Carácter transversal. Las capacidades y habilidades que hay que poner en juego en la resolución de un problema real exceden el campo acotado de cualquier área de conocimiento o disciplina. No sólo se nutren de conocimientos disciplinares científicos, sino de conocimientos procedentes de la experiencia y la observación del contexto. Son competencias con un sentido instrumental.

- Carácter dinámico/ "insaciable”. El desarrollo de las competencias no acaba nunca, precisamente porque con cada situación nueva nos ponemos 'a prueba'. Ello exige necesariamente una constante actualización y formación, constituyendo un aprendizaje a lo largo de toda la vida.

- Carácter multifuncional. Se deriva de todo lo anterior, de manera que el desarrollo de las competencias supone un aprendizaje que puede servir para resolver problemas en diferentes contextos. Ello implica la capacidad de generalizar los aprendizajes y hacer uso de ellos de maneras diversas, lo que exige que el aprendizaje sea significativo, promoviendo así una mayor reflexión y autonomía.

- Carácter básico. Las competencias que se adscriben a la denominación de básicas, responden a la habilitación que ha de adquirir el alumno para socializarse; además, constituyen referentes que facilitan la formación, a partir de Secundaria Obligatoria, en competencias preprofesionales.

\section{Similitudes entre la propuesta educativa de Rousseau y la enseñanza basada en la formación en competencias}

Por motivos de orden, se agruparán todas las similitudes, presentes en este trabajo, en torno a cuatro temas: a) el origen de ambos planteamientos, b) su finalidad, c) el método planteado y d) el cambio en la función docente. En la síntesis presentada de cada uno se podrán observar fácilmente sus semejanzas.

a) En la génesis de ambas concepciones pedagógicas, rousseauniana y socioconstructivista, se halla una semejanza. Ambos planteamientos abordan una crítica a la enseñanza del propio tiempo, coincidente en algunos puntos: excesivo verbalismo y empleo de una memoria mecánica o repetitiva sin sentido para el estudiante, que no sabe qué hacer con el conocimiento adquirido.

b) Al margen de cualquier adscripción ideológica, los diversos modos de orientar el desarrollo de la ciudadanía se vinculan a unas competencias básicas, fundamentales para la socialización. Entre esas competencias hay dos que podría suscribir Rousseau: competencia en autonomía y desarrollo personal; competencia social y ciudadana. Ambas se encuentran implícitamente latentes en Emilio y Contrato Social. La finalidad tanto de Rousseau como del vigente modelo educativo va más allá de la enseñanza de unos contenidos y transciende la formación personal individual, pensando en su utilidad social: educar buenos ciudadanos. No se puede abordar detenidamente esta cuestión por falta de espacio, pero es interesante señalarla como otra coincidencia -en cuanto a la finalidadentre Rousseau y los actuales planteamientos educativos, si bien se trata de una 
coincidencia más bien de tipo formal, pues el concepto de ciudadanía y de libertad ciudadana difieren en ambos.

c) Respecto al método, Rousseau parte de un aprendizaje a partir de la experiencia, en el que es importante la manipulación de las cosas, en un primer momento, para pasar a un aprendizaje derivado del contacto directo con problemas que surgen (o que Rousseau provoca) en el contexto inmediato, vital, de Emilio. Los contenidos no serán, como en un currículo escolar, objeto de diferentes disciplinas, sino todo aquello que sirva de utilidad para la vida del niño y, por tanto, que despierte su interés. La motivación no es extrínseca, no vendrá dada por premios ajenos al propio aprendizaje, sino a la utilidad que el alumno obtiene del mismo, lo que le sirve de acicate a su curiosidad y de único estímulo para avanzar. Por eso, no ha de aprender nada que no le sea útil o que no entienda, lo que puede considerarse un anticipo de las posteriores teorías del aprendizaje significativo y constructivista. En este planteamiento del aprendizaje cobra sentido su método de educación negativa, concebida no como una enseñanza directa, sino más bien como una protección frente al error. El aprendizaje no es fruto de la instrucción del tutor, sino de la experiencia propia del alumno dirigida discreta y hábilmente, creando condiciones favorables para el aprendizaje y evitando que pueda derivar en falsas conclusiones. Para ello, habrá que enseñarle a razonar y juzgar, lo que exigirá, primeramente, un ejercicio continuado para desarrollar bien todos los sentidos, base en la que sustentar las ideas posteriores. El planteamiento de situaciones problemáticas que Rousseau emplea en la educación de Emilio es una forma de afianzar un aprendizaje basado en la experiencia que, de otro modo, estaría desconectado de su entorno real.

Resulta llamativa la similitud con lo que, muchos años después, escriben Ausubel, Novak y Hanesian: "Tanto la resolución de problemas como la creatividad son formas de aprendizaje significativo por descubrimiento. (...) La comprensión de las condiciones del problema y la asimilación de la solución del mismo constituyen formas de aprendizaje significativo por recepción" (1983, p. 485). Estos autores distinguen entre una resolución de problemas vía discernimiento o mediante el ensayo y error, considerando significativo únicamente el aprendizaje derivado de la primera. Esto conecta de forma importante con el carácter creativo de las competencias, aludido líneas atrás. Ello no supone que el aprendizaje basado en la experiencia sea siempre significativo, pero en Rousseau adquiere connotaciones que sí son propias de este tipo de aprendizaje. La resolución de problemas exige creatividad y plantea procesos de indagación que requiere la construcción de significados y la vinculación de la nueva información con lo ya conocido. Por consiguiente, no es lo mismo aprendizaje significativo que aprendizaje basado en la experiencia, pero un aprendizaje basado en la experiencia que lleve consigo reflexión y la conexión con otras situaciones y conocimientos, tiende a adoptar los rasgos de un aprendizaje significativo.

El concepto de competencia que defiende el enfoque socioconstructivista asume la creatividad como parte esencial de la competencia, como adaptación al contexto, pero una creatividad bien entendida, esto es, soportada por un conocimiento teórico 
y no como arbitrariedad o casualidad afortunada. Por tanto, requiere combinar y ordenar los diferentes conocimientos teóricos para adecuarlos a la resolución de problemas nuevos, para los que no siempre hay protocolos o procedimientos fijos (Sierra-Arizmendiarrieta, Méndez-Giménez y Mañana-Rodríguez, 2012). Al igual que en Rousseau, la enseñanza basada en la formación en competencias también pone el acento en la relevancia de la práctica, eligiendo procedimientos inductivos, tareas y actividades con un carácter globalizador. Cobran protagonismo, así, métodos como la elaboración de Proyectos, con un carácter interdisciplinar y relacionado. Si las competencias básicas tienen un carácter holístico, contextual, transferible, reflexivo, transversal, dinámico, y funcional, el método empleado ha de favorecer en el estudiante su posible adquisición; es decir la capacidad de resolver problemas por sí solo, aplicando los conocimientos a la situación y a otros contextos similares, estimulando su curiosidad constantemente, así como reflexionando acerca de la mejor solución para cada problema. Esto implica que se le enseñe a razonar $\mathrm{y}$, por tanto, que el propio proceso de aprendizaje sea determinante. Es preciso añadir que mientras Rousseau concibe la educación de Emilio en los tres primeros libros como el sustrato sobre el cual edificar su vida social, también el carácter básico de las competencias se refiere a su función como pilar que sostenga, de modo situado, los posteriores aprendizajes.

d) En lo relativo a la función docente, Rousseau concibe que el tutor tiene como tarea principal vigilar y dirigir al aprendiz, alejándole del error 'sin que se note', con la finalidad de darle una apariencia de mayor libertad. Como parte de esa atención permanente destaca la organización de situaciones a partir de las cuales Emilio pueda aprender solo y experimentar. Dichas situaciones están orientadas a motivarle, provocando su deseo de aprender y situando a su alcance objetos de su interés, así como mostrarle diferentes caminos para que sea él mismo quien los recorra. Toda la estrategia y el despliegue de medios del tutor no sirve de nada sin la previsión acerca de cómo reaccionará Emilio, para poder reconducirle en caso necesario. En resumen, la enseñanza no ha de concebirse, para Rousseau, como instrucción directa, sino como guía en el aprendizaje, lo que supone ya una gran revolución para su época, en un doble sentido: el aprendiz es el centro del proceso $\mathrm{y}$, además, es él mismo quien toma las riendas del camino que quiere seguir. Rousseau anticipa también, de este modo, lo que constituiría más tarde, de la mano de Bruner, el aprendizaje por descubrimiento.

En la enseñanza basada en la formación en competencias, el rol del profesorado ha de transformarse en coherencia con el cambio de método que exige. Su tarea prioritaria es la organización y gestión de tareas y actividades que faciliten el desarrollo de las competencias, siendo más facilitador y guía en el aprendizaje que transmisor de contenidos. El proceso ha de discurrir como un descubrimiento guiado en el que los estudiantes elaboren y den sentido a su conocimiento a partir de las diversas situaciones-problema. Ello requiere, necesariamente, al igual que en el pensamiento de Rousseau, una atención más individualizada y un conocimiento más profundo de las capacidades y dificultades de cada estudiante. 
A continuación, se facilita una tabla de las similitudes encontradas en este trabajo entre ambos planteamientos.

\begin{tabular}{|c|c|c|c|}
\hline SIMILITUDES & $\begin{array}{l}\text { Enseñanza en Rousseau: } \\
\text { libros I-III Emilio }\end{array}$ & \multirow{5}{*}{ 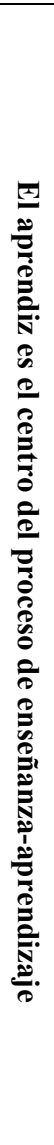 } & $\begin{array}{c}\text { Enseñanza basada en } \\
\text { formación en competencias }\end{array}$ \\
\hline ORIGEN & $\begin{array}{l}\text {-Crítica al verbalismo y a facilitar } \\
\text { contenidos ajenos a la experiencia } \\
\text { del niño } \\
\text {-Crítica memoria repetitiva y sin } \\
\text { sentido }\end{array}$ & & $\begin{array}{l}\text {-Crítica a la falta de conocimiento } \\
\text { práctico } \\
\text {-Crítica al conocimiento } \\
\text { compartimentalizado, memorístico } \\
\text { y sin sentido. }\end{array}$ \\
\hline FINALIDAD & $\begin{array}{l}\text { Ser libre en sociedad de forma } \\
\text { autosuficiente y autónoma: ser buen } \\
\text { ciudadano }\end{array}$ & & $\begin{array}{l}\text { Garantizar el desarrollo personal y } \\
\text { social, y el ejercicio de la } \\
\text { ciudadanía }\end{array}$ \\
\hline MÉTODO & $\begin{array}{l}\text { Educación negativa: } \\
\text {-no enseñanza directa y alejar del } \\
\text { error } \\
\text {-aprendizaje a partir de la experiencia, } \\
\text { enseñándole a razonar } \\
\text {-no aprender lo que no se comprenda }\end{array}$ & & $\begin{array}{l}\text { Metodología interdisciplinar: } \\
\text {-tareas y actividades globalizadoras } \\
\text {-aprendizaje basado en la resolución } \\
\text { de problemas, con reflexión y } \\
\text { creatividad } \\
\text {-fomentar aprendizaje significativo }\end{array}$ \\
\hline $\begin{array}{l}\text { FUNCIÓN } \\
\text { DOCENTE }\end{array}$ & $\begin{array}{l}\text {-Provocar situaciones de aprendizaje } \\
\text {-Enseñar a juzgar, a razonar } \\
\text {-Estimular el interés por aprender } \\
\text { basado en la necesidad y la utilidad } \\
\text {-Vigilar de cerca y guiar el proceso } \\
\text { sin que se note } \\
\text {-Conocer las posibilidades, } \\
\text { capacidades y carácter del alumno }\end{array}$ & & $\begin{array}{l}\text {-Plantear situaciones-problema } \\
\text {-Enseñar a valorar y escoger la } \\
\text { mejor solución entre varias } \\
\text { posibilidades } \\
\text {-Provocar curiosidad e interés por } \\
\text { resolver situaciones prácticas } \\
\text {-Facilitar y orientar el aprendizaje } \\
\text {-Atender de forma individualizada y } \\
\text { adaptada al progreso de cada } \\
\text { estudiante }\end{array}$ \\
\hline
\end{tabular}

Tabla I. Similitudes entre el planteamiento pedagógico de Rousseau y la enseñanza basada en la formación de competencias

Fuente: Elaboración propia

\section{Conclusión}

Llevamos ya unos años en los que se ha hecho necesario trabajar con un modelo de formación en competencias. La bibliografía es abundante y los enfoques variados. Sea cual fuere el tipo de modelo elegido, la formación en competencias no es, desde luego, la única forma de lograr un aprendizaje más comprensivo, ni tiene por qué ser mejor 
que otros modelos para el logro de la autonomía y la ciudadanía responsable, pero es el que hay que implementar en nuestro actual contexto educativo europeo. Por motivos prácticos, se hace preciso tratar de bucear, al menos, en algunos de sus antecedentes para poder comprenderlo mejor. Uno de ellos es la teoría educativa de J.J. Rousseau y, según se ha avanzado en la introducción, el objetivo prioritario de estas páginas era señalar los paralelismos existentes entre este autor y la línea socioconstructivista de formación en competencias. Las similitudes se han centrado en cuatro aspectos fundamentalmente: en el origen, la finalidad, la metodología empleada y el cambio en la función docente.

La puesta en práctica de un planteamiento educativo puede estar ligada a ideologías de cualquier signo, pero ello no es atribuible al modelo en sí, sino al uso individual o político que pueda hacerse del mismo y al tipo de sociedad que se quiera construir. En principio, y desde un punto de vista teórico, los modelos de formación en competencias con un enfoque tecnológico es más probable que puedan derivar en planteamientos de corte tecnócrata. En ellos importa prioritariamente las competencias más técnicas que reflexivas, cuya forma de adquisición se sustenta básicamente en el entrenamiento de habilidades y no en una resolución de problemas de tipo creativo. Los modelos de formación en competencias con un enfoque curricular práctico buscan un ejercicio de la ciudadanía activo. Esto iría en la línea de la teoría educativa rousseauniana que, orientada a su ideal democrático, defiende la construcción de una voluntad general que tiene que ser una voluntad formada y bien preparada para tomar decisiones sin dejarse llevar por el interés particular, advirtiendo del peligro de la olocracia como vicio o perversión de la democracia.

Dejando a un lado la polémica sobre las diversas interpretaciones existentes acerca del Contrato Social, y la distancia entre la teoría política rousseauniana y la actual en los diferentes países europeos, encontramos una idea común: la necesidad de que el aprendizaje de los futuros ciudadanos quede lejos de una adquisición automática sin sentido de contenidos y habilidades, y pase por el cedazo de la reflexión para que el conocimiento cumpla una de sus funciones más importantes: formar personas autónomas y libres. El modelo de formación en competencias y la teoría de Rousseau son sólo un medio.

\section{Referencias bibliográficas}

ARROCHA GONZÁLEZ, R. (2000). Jean Jacques Rousseau. Claves críticas de su teoría, Thémata, 25, 111-117.

AUSUBEL, D.P., NOVAK, J.D., HANESIAN, H. (1983). Psicología educativa. Un punto de vista cognoscitivo. México: Trillas.

BURGELIN, P. (1952). La philosophie de l'existence de J-J. Rousseau. Paris: Presses Universitaires de France. 
CAMARERO, J. (2010). Le rationalisme français et la théorie des émotions, Çédille. Revista de estudios franceses, 6, 52-72.

DAM, G., GEIJSEL, F., REUMERMAN, R. Y LEDOUX, G. (2011). Measuring Young People's Citizenship Competences. European Journal of Education, 46 (3), 354-372.

DOMINGO SEGOVIA, J. Y BARRERO FERNÁNDEZ, B. (2010). Competencias básicas y aprendizajes imprescindibles, en Moral Santaella, C. (Coord.) Didáctica. Teoría y práctica de la enseñanza. Madrid: Pirámide, 111-125.

ESCAMILLA, A. (2008). Las competencias básicas. Claves para su desarrollo en los centros. Barcelona: Graó.

FEDI, L. (2011). Les paradoxes éducatifs de Rousseau, Revue Philosophique, 4, 487506.

GOÑI, J. M. (2009). El desarrollo de la competencia matemática en el currículo escolar de la Educación Básica. Educatio Siglo XXI, 27 (1), 33-58.

GUENARD, F. (2009). Devenir sociable, devenir citoyen. Émile dans le monde. Archives de Philosophie, 72 (1), 9-29.

GUTIÉRREZ ZULOAGA, I. (2002). Modelos educativos paradigmáticos en la Historia de la Educación, Arbor, 173 (681), 3-17.

HALÁSZ, G. Y MICHEL, A. (2011). Key Competences in Europe: Interpretation, Policy Formulation and Implementation, European Journal of Education, 46 (3), 289-306.

HANLEY, R.P. (2012). Rousseau's Virtue Epistemology. Journal of the History of Philosophy, 50 (2), 239-263.

HANNOUN, H. (1979). L'Éducation naturelle. Paris: Presses Universitaires de France.

KONTIO, K. (2003). The Idea of Autarchy in Rousseau's Natural Education: recovering the natural harmony? Scandinavian Journal of Educational Research, 47 (1), 3-19.

MÉNDEZ-GIMÉNEZ, A., Sierra-Arizmendiarrieta, B. y Mañana-Rodríguez, J. (2013). Percepciones y creencias de los docentes de Primaria del Principado de Asturias sobre las competencias básicas. Revista de Educación, 362, 737-761.

MOYA OTERO, J. (2008). Las competencias básicas en el diseño y el desarrollo del currículo. Revista Qurriculum, 21, 57-78.

OELKERS, J. (2002). Rousseau and the image of 'modern education'. Curriculum Studies, 34 (6), 679-698.

PEPPER, D. (2011). Assessing Key Competences across the Curriculum and Europe. European Journal of Education, 46 (3), 335-353. 
PÉREZ FERRA, M. (2011). Capacidades docentes del profesor universitario para la gestión de un currículum orientado a la diversidad, en Pantoja, A.; Zwierewicz, M. y Moraes, R. (Coods). Diversidad y adversidad en Educación. Jaén: Joxman, 58-81.

PERRENOUD, P. (2004). Desarrollar la práctica reflexiva en el oficio de enseñar. Barcelona: Graó.

RODRÍGUEZ TORRES, J. (2010). De las programaciones didácticas a la unidad didáctica: incorporación de competencias básicas y la concreción de tareas. Revista Docencia e Investigación, 20, 245-270.

ROMANI, S. Y RAJOBAC, R. (2011). Iluminismo pedagógico: educação e adolescencia no Livro III do Emílio de Rousseau, Revista Espaço Acadêmico, 125, 103-109.

ROUSSEAU, J-J. (1959-95). Oeuvres Complètes [OC], en Gagnebin, B. y Raymond, M. (Eds.) (cinco volúmenes). Paris: Bibliothêque de la Pléiade, Gallimard.

SÁNCHEZ SANTAMARÍA, J. (2012). Del concepto a las estructuras: implicaciones organizativas del enfoque de competencias en educación, Bordón, 64 (1), 127-140.

SIERRA-ARIZMENDIARRIETA, B. (1997). Dos formas de libertad en J-J. Rousseau. Pamplona: Eunsa.

SIERRA-ARIZMENDIARRIETA, B.; Méndez-Giménez, A. y Mañana-Rodríguez, J. (2012). Necesidad y propuesta de un procedimiento para programar por Competencias Básicas, Aula Abierta, 40 (3), 33-46.

SIERRA-ARIZMENDIARRIETA, B.; Méndez-Giménez, A.; Mañana-Rodríguez, J. (2013). La programación por competencias básicas: hacia un cambio metodológico interdisciplinar, Revista Complutense de Educación, 24 (1), 165-184.

SIMÕES FRANCISCO, M. de F. (2000). La filosofía de la educación de Rousseau. Una propuesta de relectura del Emilio. Revista Educación y Pedagogía, 12 (26-27), 65-75.

TIANA, A.; MOYA, J. Y LUENGO, F. (2011). Implementing Key Competences in Basic Education: reflections on curriculum design and development in Spain, European Journal of Education, 46 (3), 307-322.

VISO ALONSO, J.R. (2010). Qué son las competencias. Vol. 1. Madrid: EOS.

WENDT, C.E. Y DALBOSC, C.A. (2012). Iluminismo pedagógico e educação natural em Jean-Jacques Rousseau, Revista Educação, 37 (2), 229-240.

ZABALA, A. Y ARNAU, L. (2007). Cómo aprender y enseñar competencias. Barcelona: Graó.

\section{Referencias digitales}

OCDE (2005). La definición y selección de competencias clave. Resumen ejecutivo. Recuperado de http://www.deseco.admin.ch/bfs/deseco/en/index/03/02.parsys.78532.downloadList 
.94248.DownloadFile.tmp/2005.dscexecutivesummary.sp.pdf (Consultado 19 de junio 2013).

GORdON, J., HALÁSZ, G., KRAWCZYK, M., LENEY, T., MICHEL, A. PEPPER, D., PUTKIEWICZ, E., WISNIEWSKI, J. (2009). Key Competences in Europe: Opening Doors for Lifelong Learns across the School Curriculum and Teacher Education. Warsaw (Polonia): CASE (Center for Social and Economic Research). Recuperado

de http://papers.ssrn.com/sol3/papers.cfm?abstract_id=1517804 (Consultado 4 de septiembre de 2013).

MULDER, M., WEIGEL, T. Y COLLINGS, K. (2008). El concepto de competencia en el desarrollo de la educación y formación profesional en determinados países miembros de la U.E.: un análisis crítico. Profesorado. Revista de currículum y formación del profesorado, 12(3), 1-25. http://www.ugr.es/ recfpro/rev123ART6.pdf (consultado el 12 de septiembre de 2013)

\section{Correspondencia con los autores}

Beatriz SIERRA-ARIZMENDIARRIETA.

Facultad de Formación del Profesorado y Educación.

Universidad de Oviedo. C/Aniceto Sela, s/n. 33005-Oviedo.

Teléfono: 985102859

e-mail: bsierra@uniovi.es

Miguel PÉREZ FERRA.

Facultad de Humanidades y Ciencias de la Educación.

Universidad de Jaén. Departamento de Pedagogía, edf ${ }^{\circ}$ C-5, dependencia 247.

Campus de Las Lagunillas, s/n 23071. Jaén.

Teléfono: 953211805

e-mail:mperez@ujaen.es 